Article

\title{
Another Note on Paraconsistent Neutrosophic Sets
}

\author{
Francisco Gallego Lupiáñez
}

Department of Mathematics, Universidad Complutense, 28040 Madrid, Spain; fg_lupianez@mat.ucm.es

Received: 14 June 2017; Accepted: 27 July 2017; Published: 2 August 2017

\begin{abstract}
In an earlier paper, we proved that Smarandache's definition of neutrosophic paraconsistent topology is neither a generalization of Çoker's intuitionistic fuzzy topology nor a generalization of Smarandache's neutrosophic topology. Recently, Salama and Alblowi proposed a new definition of neutrosophic topology, that generalizes Çoker's intuitionistic fuzzy topology. Here, we study this new definition and its relation to Smarandache's paraconsistent neutrosophic sets.
\end{abstract}

Keywords: logic; set-theory; topology; Atanassov's intuitionistic fuzzy sets

\section{Introduction}

In various papers, Smarandache [1,2] has generalized Atanassov's intuitionistic fuzzy sets [3] to neutrosophic sets.

Çoker [4] defined and studied intuitionistic fuzzy topological spaces.

On the other hand, various authors including Priest et al. [5] worked on paraconsistent logic, that is, logic where some contradiction is admissible. We refer the reader to studies of References [6-8] as well as the work on paraconsistent fuzzy logic conducted in Reference [9].

Smarandache [2] also defined neutrosophic paraconsistent sets, and proposed a natural definition of neutrosophic paraconsistent topology.

In an earlier paper [10], we proved that this Smarandache's definition of neutrosophic paraconsistent topology is neither a generalization of Çoker's intuitionistic fuzzy topology nor of Smarandache's general neutrosophic topology.

Recently, Salama and Alblowi [11] proposed a new definition of neutrosophic topology that generalizes Çoker's intuitionistic fuzzy topology.

In this paper, we study this new definition and its relation to Smarandache's paraconsistent neutrosophic sets.

The interest of neutrosophic paraconsistent topology was previously shown by us [12] (Section 4).

\section{Materials and Methods}

First, we present some basic definitions:

Robinson [13] developed the non-standard analysis, a formalization of analysis and a branch of mathematical logic, which rigorously defines infinitesimals. Formally, a number $\mathrm{x}$ is said to be infinitesimal if for all positive integers $\mathrm{n}$, one has $|x|<1 / \mathrm{n}$. Let $\varepsilon \geq 0$ be such an infinitesimal number. The hyper-real number set is an extension of the real number set, which includes classes of infinite numbers and classes of infinitesimal numbers. Let us consider the non-standard finite numbers $(1+)=1+\varepsilon$, where " 1 " is its standard part and " $\varepsilon$ " its non-standard part, and $(-0)=0-\varepsilon$, where " 0 " is its standard part and " $\varepsilon$ " its non-standard part. Then, we denote $]-0,1+[$ to indicate a non-standard unit interval. Obviously, 0 and 1 , and analogously non-standard numbers infinitely smaller but less than 0 or infinitely smaller but greater than 1 , belong to the non-standard unit interval. It can be proven that $S$ is a standard finite set if and only if every element of $S$ is standard (See Reference [14]). 
Definition 1. In Reference [2], let T,I,F be real standard or non-standard subsets of the non-standard unit interval $]-0,1+[$, with

$\sup T=t_{\text {sup }} \inf T=t_{\text {inf }}$

$\sup I=i_{\text {sup }}, \inf I=i_{\text {inf }}$;

$\sup F=f_{\text {sup }}, \inf F=f_{\text {inf }}$ and

$n_{\text {sup }}=t_{\text {sup }}+i_{\text {sup }}+f_{\text {sup }}, n_{\text {inf }}=t_{\text {inf }}+i_{\text {inf }}+f_{\text {inf }}$.

T, I, F are called neutrosophic components. Let $U$ be a universe of discourse, and $M$ a set included in $U$. An element $x$ from $U$ is noted with respect to the set $M$ as $x(T, I, F)$ and belongs to $M$ in the following way: it is $t \%$ true in the set, $i \%$ indeterminate (unknown if it is) in the set, and $f \%$ false, where $t$ varies in $T$, $i$ varies in I, fvaries in $F$. The set $M$ is called a neutrosophic set (NS).

Definition 2. In Reference [2], a neutrosophic set $x(T, I, F)$ is called paraconsistent, if $\inf (T)+\inf (I)+\inf (F)>1$.

Definition 3. In Reference [11], the NSs $0_{N}$ and $1_{N}$ are defined as follows:

$0_{N}$ may be defined as:

$$
\begin{array}{ll}
\left(0_{1}\right) & 0_{N}=x(0,0,1) \\
\left(0_{2}\right) & 0_{N}=x(0,1,1) \\
\left(0_{3}\right) & 0_{N}=x(0,1,0) \\
\left(0_{4}\right) & 0_{N}=x(0,0,0)
\end{array}
$$

$1_{N}$ may be defined as:

$$
\begin{array}{ll}
\left(1_{1}\right) & 1_{N}=x(1,0,0) \\
\left(1_{2}\right) & 1_{N}=x(1,0,1) \\
\left(1_{3}\right) & 1_{N}=x(1,1,0) \\
\left(1_{4}\right) & 1_{N}=x(1,1,1)
\end{array}
$$

Definition 4. In Reference [11], let $X$ be a non-empty set and $A=x\left(T_{A}, I_{A}, F_{A}\right), B=x\left(T_{B}, I_{B}, F_{B}\right)$ be NSs. Then:

$A \cap B$ may be defined as:

$$
\begin{array}{ll}
\left(I_{1}\right) & A \cap B=x\left(T_{A} \cdot T_{B}, I_{A} \cdot I_{B}, F_{A} \cdot F_{B}\right) \\
\left(I_{2}\right) & A \cap B=x\left(T_{A} \wedge T_{B}, I_{A} \wedge I_{B}, F_{A} \vee F_{B}\right) \\
\left(I_{3}\right) & A \cap B=x\left(T_{A} \wedge T_{B}, I_{A} \vee I_{B}, F_{A} \vee F_{B}\right)
\end{array}
$$

$A \cup B$ may be defined as:

$\left(U_{1}\right) \quad A \cup B=x\left(T_{A} \vee T_{B}, I_{A} \vee I_{B}, F_{A} \wedge F_{B}\right)$

(U⿱ $\left.U_{2}\right) \quad A \cup B=x\left(T_{A} \vee T_{B}, I_{A} \wedge I_{B}, F_{A} \wedge F_{B}\right)$

Definition 5. In Reference [11], let $\left\{A_{j} \mid j \in J\right\}$ be an arbitrary family of NSs in $X$, then:

(1) $\cap A_{j}$ may be defined as:

$$
\begin{aligned}
& \text { (i) } \cap A_{j}=x(\wedge, \wedge, \vee) \\
& \text { (ii) } \cap A_{j}=x(\wedge, \vee, \vee)
\end{aligned}
$$

(2) $\cup A_{j}$ may be defined as:

$$
\begin{aligned}
& \text { (i) } \cup A_{j}=x(\vee, \vee, \wedge) \\
& \text { (ii) } \cup A_{j}=x(\vee, \wedge, \wedge)
\end{aligned}
$$


Definition 6. In Reference [11], a neutrosophic topology on a non-empty set X is a family $\tau$ of NSs in X satisfying the following properties:

(1) $0_{N}$ and $1_{N} \in \tau_{\tau}$;

(2) $G_{1} \cap G_{2} \in \tau$, for any $G_{1}, G_{2} \in \tau$;

(3) $\cup G_{j} \in \tau$ or any subfamily $\left\{G_{j}\right\}_{j \in J}$ of $\tau$.

In this case, the pair $(\mathbf{X}, \boldsymbol{\tau})$ is called a neutrosophic topological space.

\section{Results}

Proposition 1. The set of paraconsistent NSs with the definitions above is not a bounded lattice.

\section{Proof.}

(1) It is necessary to omit a definition of $\cap$, because we will need $\cap$ of paraconsistent NSs to be paraconsistent. Indeed, let $\mathbf{A}=x(1 / 2,1 / 2,1 / 2)$ and $B=x(1 / 2,1 / 3,1 / 3)$ (both are paraconsistent NSs), but $1 / 4+1 / 6+1 / 6$ is not $>1$. Then, the case with product $\left(\left(\mathrm{I}_{1}\right)\right.$, in Definition 4$)$ must be deleted for paraconsistent NSs.

(2) The definitions of $0_{N}$ and $1_{N}$ also have problems for paraconsistent NSs:

(a) Only $\left(0_{2}\right)$ and $\left(1_{2}\right),\left(1_{3}\right),\left(1_{4}\right)$ are paraconsistent;

(b) If we want all NSs: $0_{N} \cup 0_{N}, 0_{N} \cup 1_{N}, 1_{N} \cup 1_{N}, 0_{N} \cap 0_{N}$, and $0_{N} \cap 1_{N}$ to be paraconsistent NSs, it is necessary to delete $1_{2}$ in Definition 3 , because with this definition,

$0_{\mathrm{N}} \cap 1_{\mathrm{N}}$ is equal either to $x(0,0,1)$ which is not paraconsistent, or to $x(0,1,1)=0_{\mathrm{N}}$.

The other cases have no problems: $0_{N} \cup 0_{N}=x(0,1,1)=0_{N}$,

$0_{N} \cup 1_{N}$ is equal either to $x(1,0,1)$, or to $x(1,1,0)$, or $x(1,1,1)$, i.e equal to $1_{N}$, $1_{N} \cup 1_{N}$ is equal either to $x(1,0,1)$, or to $x(1,1,0)$, or $x(1,1,1)$, i.e equal to $1_{N}$, $0_{\mathrm{N}} \cap 0_{\mathrm{N}}=\mathrm{x}(0,1,1)=0_{\mathrm{N}}$,

$1_{N} \cap 1_{N}$ is equal either to $x(1,0,1)$, or to $x(1,1,0)$, or $x(1,1,1)$, i.e equal to $1_{N}$.

Then, after these changes in Definitions 3 and 4, Definition 6 is suitable for Smarandache's paraconsistent NSs, and one can work on paraconsistent neutrosophic topological spaces.

Definition 7. Let X be a non-empty set. A family $\tau$ of neutrosophic paraconsistent sets in $X$ will be called a paraconsistent neutrosophic topology if:

(1) $0_{N}=x(0,1,1)$, and $1_{N}=x(1,1,0)$ or $x(1,1,1)$, are in $\tau$;

(2) $G_{1} \cap G_{2} \in \tau$ for any $G_{1}, G_{2} \in \tau$ (where $\cap$ is defined by $\left(I_{2}\right)$ or $\left(I_{3}\right)$ );

(3) $\cup G_{j} \in \tau$ for any subfamily $\left\{G_{j}\right\}_{j \in J}$ of $\tau$ (where $\cup$ is defined by Definition 5).

In this case, the pair $(X, \tau)$ is called a paraconsistent neutrosophic topological space.

Remark. The above notion of paraconsistent neutrosophic topology generalizes Çoker's intuitionistic fuzzy topology when all sets are paraconsistent.

\section{Discussion}

Definition 7 is suitable for the work on paraconsistent neutrosophic topological spaces. In fact:

Proposition 2. The set of paraconsistent NSs with the following definitions,

(a) $\quad 0_{N}=x(0,1,1)$, and $1_{N}=x(1,1,0)$ or $x(1,1,1)$

(b) $\cap$ defined by $\left(I_{2}\right)$ or $\left(I_{3}\right)$

(c) $\cup$ defined by Definition 5 is a bounded lattice.

Proof. Obvious from proof of Proposition 1. 


\section{References}

1. Smarandache, F. A unifying field in Logics: Neutrosophic Logic. Mult.-Valued Log. 2002, 8, 385-438.

2. Smarandache, F. Neutrosophic set-A generalization of the intuitionistic fuzzy set. Intern. J. Pure Appl. Math. 2005, 24, 287-297.

3. Atanassov, K.T. Intuitionistic fuzzy sets. Fuzzy Sets Syst. 1986, 20, 87-96. [CrossRef]

4. Çoker, D. An introduction to intuitionistic fuzzy topological spaces. Fuzzy Sets Syst. 1997, 88, 81-89. [CrossRef]

5. Priest, G.; Routley, R.; Norman, J. (Eds.) Paraconsistent Logic: Essays on the Inconsistent; Philosophia Verlag: Munich, Germany, 1989.

6. Beziau, I.Y.; Chakraborty, M.; Dutta, D. New directions in paraconsistent logic. In Proceedings of the 5th World Congress on Paraconsistency (WCP), Kolkata, India, 13-17 February 2014; Springer: Kolkata, 2014.

7. Da Costa, N.C.A. Nota sobre o conceito de contradição. Anuário Soc. Paranense Mat. 1958, 1, 6-8.

8. Peña, L. Dialectical arguments, matters of degree, and paraconsistent Logic. In Argumentation: Perspectives and Approaches; van Eemeren, F.H., Grootendorst, R., Blair, J.A., Willard, C.A., Eds.; Foris Publication: Dordrecht, The Netherlands, 1987; pp. 426-433.

9. Avron, A. Paraconsistent fuzzy logic preserving non-falsity. Fuzzy Sets Syst. 2016, 292, 75-84. [CrossRef]

10. Lupiáñez, F.G. On Neutrosophic Paraconsistent Topology. Kybernetes 2010, 39, 598-601. [CrossRef]

11. Salama, A.A.; Alblowi, S.A. Neutrosophic set and neutrosophic topological spaces. IOSR J. Math. 2012, 3, 31-35. [CrossRef]

12. Lupiáñez, F.G. On neutrosophic sets and topology. In New Trends in Neutrosophic Theory and Applications; Smarandache, F., Pramanik, S., Eds.; Pons Editions: Brussels, Belgium, 2016; pp. 305-313.

13. Robinson, A. Non-Standard Analysis; North Holland: Amsterdam, The Netherlands, 1966.

14. Hrbacek, K. Nonstandard Set Theory. Am. Math. Mon. 1979, 86, 659-677. [CrossRef]

(C) 2017 by the author. Licensee MDPI, Basel, Switzerland. This article is an open access article distributed under the terms and conditions of the Creative Commons Attribution (CC BY) license (http://creativecommons.org/licenses/by/4.0/). 